\title{
Direct Observation of Coherent Interorbital Spin-Exchange Dynamics
}

\author{
G. Cappellini, ${ }^{1,3}$ M. Mancini, ${ }^{2,3}$ G. Pagano, ${ }^{4,3}$ P. Lombardi, ${ }^{1}$ L. Livi, ${ }^{2}$ M. Siciliani de Cumis, ${ }^{3}$ P. Cancio, ${ }^{3,1,2}$ M. Pizzocaro, ${ }^{5}$ \\ D. Calonico, ${ }^{5}$ F. Levi, ${ }^{5}$ C. Sias, ${ }^{3,1}$ J. Catani, ${ }^{3,1}$ M. Inguscio, ${ }^{5,2,1}$ and L. Fallani ${ }^{2,1,3, *}$ \\ ${ }^{1}$ LENS European Laboratory for Nonlinear Spectroscopy, Sesto Fiorentino 50019, Italy \\ ${ }^{2}$ Department of Physics and Astronomy, University of Florence, Sesto Fiorentino 50019, Italy \\ ${ }^{3}$ INO-CNR Istituto Nazionale di Ottica del CNR, Sezione di Sesto Fiorentino, Sesto Fiorentino 50019, Italy \\ ${ }^{4}$ Scuola Normale Superiore di Pisa, Pisa 56126, Italy \\ ${ }^{5}$ INRIM Istituto Nazionale di Ricerca Metrologica, Torino 10135, Italy
}

(Received 25 June 2014; published 15 September 2014)

\begin{abstract}
We report on the first direct observation of fast spin-exchange coherent oscillations between different long-lived electronic orbitals of ultracold ${ }^{173} \mathrm{Yb}$ fermions. We measure, in a model-independent way, the strength of the exchange interaction driving this coherent process. This observation allows us to retrieve important information on the interorbital collisional properties of ${ }^{173} \mathrm{Yb}$ atoms and paves the way to novel quantum simulations of paradigmatic models of two-orbital quantum magnetism.
\end{abstract}

PACS numbers: 03.75.Ss, 34.50.Cx, 37.10.Jk, 67.85.Lm

Alkaline-earth-like (AEL) atoms are providing a new valuable experimental platform for advancing the possibilities of quantum simulation with ultracold gases [1]. For instance, the purely nuclear spin of ground-state AEL fermionic isotopes results in the independence of the atom-atom scattering properties from the nuclear spin projection. This feature has enabled the investigation of multicomponent ${ }^{173} \mathrm{Yb}$ fermions with $\mathrm{SU}(N)$ interaction symmetry both in optical lattices [2] and in onedimensional quantum wires [3]. In addition to their nuclear spin, AEL atoms offer experimental access to supplementary degrees of freedom, in particular, to a long-lived electronically excited state $|e\rangle=\left|{ }^{3} P_{0}\right\rangle$ which can be coherently populated from the ground state $|g\rangle=\left|{ }^{1} S_{0}\right\rangle$ by optical excitation on an ultranarrow clock transition. The possibility of coherently manipulating both the orbital and the spin degree of freedom has recently been envisioned to grant the realization of paradigmatic models of two-orbital magnetism, like the Kondo model [4]. In this context, the two electronic states $|g\rangle$ and $|e\rangle$ play the roles of two different orbitals.

Recent experiments have investigated the $\mathrm{SU}(N)$ symmetry in $|g\rangle-|e\rangle$ ultracold collisions of two-electron atoms [5] and reported on first signatures of spin-exchange interactions between atoms in the two electronic states [6]. Spin-exchange interactions arise from the difference in the spin-singlet and spin-triplet potential curves in the scattering of one $|g\rangle$ and one $|e\rangle$ atom. Let us assume that the two interacting atoms are in different nuclear spin states $|\uparrow\rangle$ and $|\downarrow\rangle$ (where the arrows are placeholders for two arbitrary nuclear spin states) and that they share the same spatial wave function. At zero magnetic field, the degeneracy of the configurations $|g \uparrow, e \downarrow\rangle$ and $|g \downarrow, e \uparrow\rangle$, which are associated with a well-defined spin in each orbital [7], is lifted by the atom-atom interaction and the eigenstates are the orbital-symmetric (spin-singlet) $\left|e g^{+}\right\rangle$and the orbitalantisymmetric (spin-triplet) $\left|e g^{-}\right\rangle$states [4]

$$
\left|e g^{ \pm}\right\rangle=\frac{1}{\sqrt{2}}(|g \uparrow, e \downarrow\rangle \mp|g \downarrow, e \uparrow\rangle) .
$$

(a)
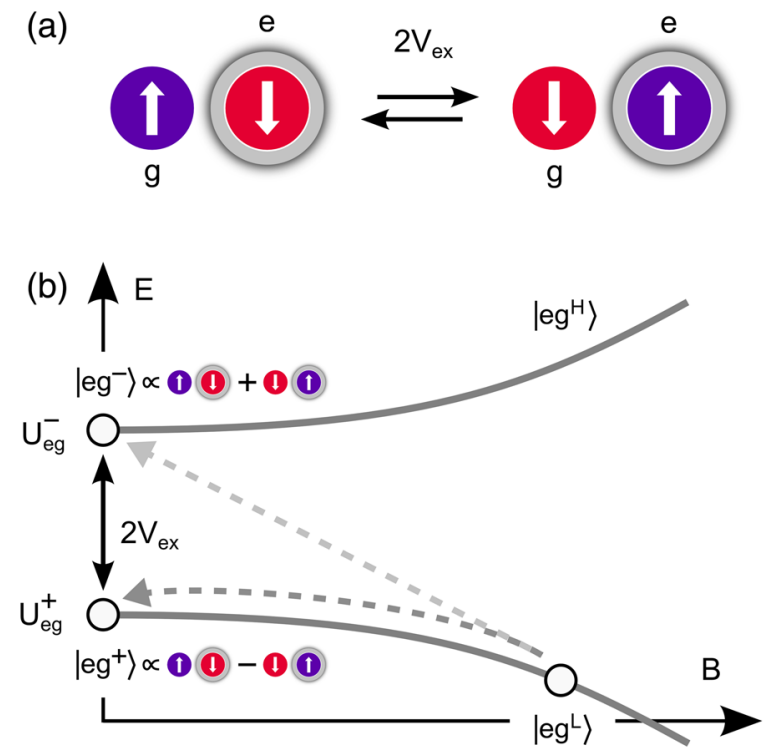

FIG. 1 (color online). Two-orbital spin-exchange interaction in AEL atoms. (a) One atom in the ground state $|g\rangle$ and one atom in the long-lived electronic state $|e\rangle$ periodically "exchange" their nuclear spins because of the different interaction energy in the spin-singlet $\left|e g^{+}\right\rangle$and spin-triplet $\left|e g^{-}\right\rangle$two-particle states (note that in the graphical notation, the two-particle exchange symmetry is implicit [7]). (b) Dependence of the two-particle energy on the magnetic field $B$. The spin dynamics is initiated by exciting the two atoms to the $\left|e g^{L}\right\rangle$ state at finite $B$ and then quenching the magnetic field to a small bias value in order to create a superposition of the $\left|e g^{+}\right\rangle$and $\left|e g^{-}\right\rangle$states (dashed arrows). 
Owing to the different atom-atom scattering properties, these two states have different interaction energies $U_{\text {eg }}^{ \pm}$, as sketched in Fig. 1. Preparing the two atoms in the initial state $\left|\psi_{0}\right\rangle=|g \uparrow, e \downarrow\rangle=1 / \sqrt{2}\left[\left|e g^{+}\right\rangle+\left|e g^{-}\right\rangle\right]$would result in a spin-exchange dynamics in which the spins of the $|g\rangle$ and $|e\rangle$ atoms are periodically flipped at a frequency $2 V_{\mathrm{ex}} / h=\left|U_{e g}^{-}-U_{e g}^{+}\right| / h$, with the probability of finding a ground-state atom in the $|g \uparrow\rangle$ state being given by

$$
P(|g \uparrow\rangle)(t)=\frac{1}{2}\left[1+\cos \left(\frac{2 V_{\mathrm{ex}}}{\hbar} t\right)\right] .
$$

Recent measurements have suggested that in ${ }^{173} \mathrm{Yb}$, the scattering lengths associated with the spin-triplet and spin-singlet scatterings are quite different [6], resulting in a large interorbital spin-exchange interaction energy $V_{\text {ex }}$. However, spin oscillations induced by such an interaction have not been observed, and no demonstration of the coherence of this exchange process has been shown. Here, we report on the first, time-resolved observation of interorbital spin oscillations. This measurement clearly demonstrates the coherent nature of the exchange interaction, which is fundamentally important for its applications in quantum simulation. By measuring the oscillation frequency, we determine the interaction strength $V_{\mathrm{ex}}$ in a model-independent way, finding it to be much larger than both the Fermi energy $E_{F}=k_{B} T_{F}$ and $k_{B} T$ (where $k_{B}, T_{F}$, and $T$ are the Boltzmann constant, the Fermi temperature, and the gas temperature, respectively). Moreover, our measurements allow us to determine the scattering length associated with the antisymmetric scattering potential.

The experiment is performed on quantum degenerate Fermi gases of ${ }^{173} \mathrm{Yb}$ in a balanced mixture of two different states out of the $I=5 / 2$ nuclear spin manifold $\mid m_{I}=$ $+5 / 2\rangle \equiv|\uparrow\rangle$ and $\left|m_{I}=-5 / 2\right\rangle \equiv|\downarrow\rangle$. The atoms, at an initial temperature $T \simeq 0.15 T_{F} \simeq 25 \mathrm{nK}$, are trapped in a $3 \mathrm{D}$ optical lattice, with a variable depth up to $s=40$, where $s$ measures the lattice depth in units of the recoil energy $E_{R}=h^{2} / 2 m \lambda_{L}^{2}, \lambda_{L}$ and $m$ being the lattice wavelength and atomic mass, respectively. In our experimental conditions (see the Supplemental Material [8]), the site occupancy in the center of the trap is $n \simeq 1$ for each spin state. The longlived $|e\rangle$ state is populated by exciting the ${ }^{1} S_{0} \rightarrow{ }^{3} P_{0}$ intercombination transition with linearly polarized light coming from a $\lambda=578 \mathrm{~nm}$ ultranarrow laser stabilized to an ultralow-expansion glass optical resonator with a closedloop linewidth below $10 \mathrm{~Hz}$ [11]. The lattice is operating at the magic wavelength $\lambda_{L}=759.35 \mathrm{~nm}$, which is not shifting the ${ }^{1} S_{0} \rightarrow{ }^{3} P_{0}$ transition frequency [12].

A typical spectrum for a long excitation time $(\simeq 100 \mathrm{~ms})$ is reported in Fig. 2(a), showing the presence of several peaks associated with the excitations of both singly and doubly occupied sites. We are able to spectroscopically distinguish the different peaks and address only the doubly occupied sites by adding a static, uniform magnetic field $B$ (which was set to $28 \mathrm{G}$ for the data shown in the figure).

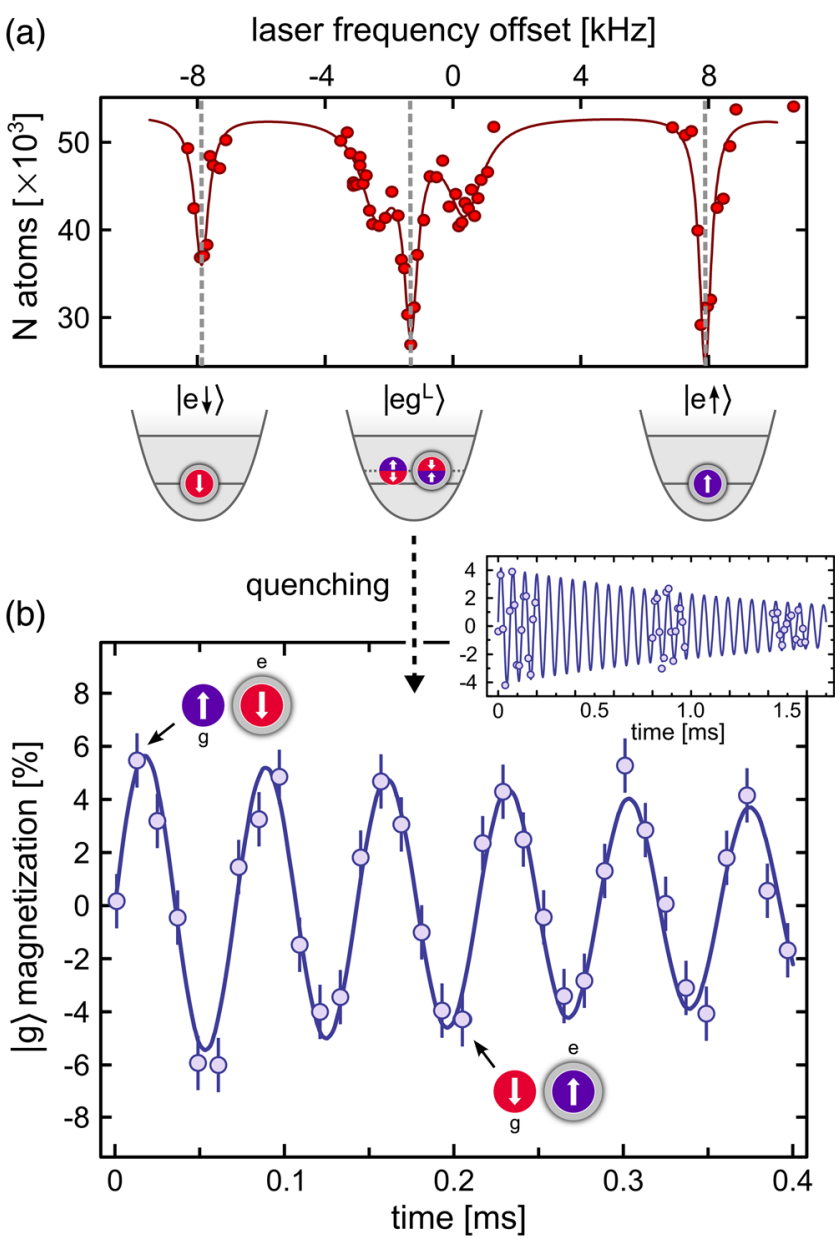

FIG. 2 (color online). (a) Spectrum of the $\lambda=578 \mathrm{~nm}$ clock transition for the excitation of a two-spin mixture of ${ }^{173} \mathrm{Yb}$ atoms trapped in a 3D lattice. The vertical axis shows the number of residual $|g\rangle$ atoms after the excitation, while the horizontal axis shows the offset with respect to the clock transition frequency. The labels below the plot indicate the most prominent features of the spectrum. The dependence of the peak centers on the magnetic field $B$ allows us to attribute them to the excitation of one atom in either singly occupied sites $\left(|e \downarrow\rangle\right.$ and $|e \uparrow\rangle$ ) or in doubly occupied sites ( $\left|e g^{L}\right\rangle$ ) (see Ref. [6] for the assignment of the other peaks). (b) Time-resolved detection of spin-exchange oscillations. The points show the difference in fractional population between $|g \uparrow\rangle$ and $|g \downarrow\rangle$ atoms. The data shown in the figure have been taken at a lattice depth $s=30.8$ after quenching the magnetic field from $60 \mathrm{G}$ to a bias field of $3.5 \mathrm{G}$. The points have been offset by a constant value $(\simeq 5 \%)$ to take into account a slight unbalance of the spin mixture resulting from an imperfect preparation of the initial state [which also leads to the asymmetry of the $|e \downarrow\rangle$ and $|e \uparrow\rangle$ peaks in (a)]. The points are averages over five repeated measurements, and the line is the result of a fit with a damped sinusoidal function (a global error bar based on the fit residuals has been assigned to the points). The inset shows a different data set taken at $s=35$ with oscillations extending to longer times.

Because of the Zeeman shift, at a finite $B$, the eigenstates of the system become an admixture of spin-singlet and spintriplet states $\left|e g^{L}\right\rangle=\alpha\left|e g^{+}\right\rangle+\beta\left|e g^{-}\right\rangle\left(\left|e g^{H}\right\rangle=\beta^{*}\left|e g^{+}\right\rangle-\right.$ $\alpha^{*}\left|e g^{-}\right\rangle$), with $|\alpha|^{2}=|\beta|^{2}=1 / 2$ for infinitely large magnetic fields [6] (see also Fig. 1). Note that at $B=0$, 
the $\left|e g^{H}\right\rangle$ state reduces to the spin-triplet state $\left|e g^{-}\right\rangle$, which cannot be populated by direct laser excitation since the ground state is a spin singlet.

In order to initiate the spin dynamics, we first excite the atoms with a $\pi$ pulse resonant with the $\left|e g^{L}\right\rangle$ excitation frequency. The excitation is performed at a large lattice depth $s_{\text {in }} \geq 30$, in order to avoid tunneling of atoms during the excitation time, and at large magnetic field $(60 \mathrm{G})$, in order to have a sizeable admixture of the spin-triplet state $\left|e g^{-}\right\rangle$into the $\left|e g^{L}\right\rangle$ state $\left(|\alpha|^{2} \simeq 0.75,|\beta|^{2} \simeq 0.25\right)$. Then, we rapidly decrease the magnetic field to a very low bias field $(3.5 \mathrm{G})$ in a time $t_{\text {ramp }}=25 \mu \mathrm{s}$, which is fast enough to have a significant population of the $\left|e g^{H}\right\rangle \simeq\left|e g^{-}\right\rangle$state by nonadiabatic Landau-Zener excitation (see dashed arrows in Fig. 1) [13]. The creation of a superposition of $\left|e g^{-}\right\rangle$and $\left|e g^{+}\right\rangle$states allows us to start the spin dynamics, which is observed by detecting the fraction of ground-state atoms in the different spin states by performing optical Stern-Gerlach detection after different evolution times [14]. Figure 2(b) shows clear oscillations of the ground-state magnetization $[N(g \uparrow)-N(g \downarrow)] /[N(g \uparrow)+N(g \downarrow)]$, which are driven by the spin-exchange process. These oscillations, clearly visible for tens of periods (as shown in the inset), provide a clear demonstration of the coherent nature of this spin-exchange interaction. The measurement of their frequency provides a direct, model-independent determination of the interaction strength, which is $2 V_{\mathrm{ex}}=h \times(13.87 \pm$ $0.17) \mathrm{kHz}$ for the data in Fig. 2(b), taken at $s=30.8$ [15].

We note that the relatively small amplitude of the oscillation in Fig. 2(b) can be ascribed to three different causes: (1) a small initial admixture of the $\left|e g^{-}\right\rangle$state in the $\left|e g^{L}\right\rangle$ state (due to excitation at a finite $B$ ), (2) the finite switching time of the magnetic field, which makes the projection onto the new eigenstates at low $B$ only partially diabatic, and (3) the presence of singly occupied lattice sites not participating in the spin oscillation yet contributing to the background signal. We also have checked that these spin oscillations disappear if no laser excitation pulse is performed: collisions among $|g\rangle$ atoms can only take place in the spin-singlet channel, and the strong $\mathrm{SU}(N)$ interaction symmetry grants the absence of spin-changing collisions [3]. We have also checked that no other nuclear spin states, different from $|\uparrow\rangle$ and $|\downarrow\rangle$, are populated during the spin-exchange dynamics.

In order to quantify the strength and the properties of the interorbital exchange interaction, we have measured the frequency of these spin oscillations as a function of the lattice depth $s$ and of the magnetic field $B$.

The points in Fig. 3(a) show the dependence of the spinoscillation frequency $2 V_{\mathrm{ex}} / h$ on the lattice depth, clearly exhibiting a monotonic increase with $s$. In these measurements, the optical excitation is performed at a lattice depth $s_{\text {in }} \geq 30$; then, the optical lattice is ramped to $s$ in $\sim 700 \mu \mathrm{s}$, immediately before the quench which initiates the spin dynamics. The measured values of $2 V_{\text {ex }}$ are significantly large, $\approx 5$ times larger than the Hubbard interaction energy

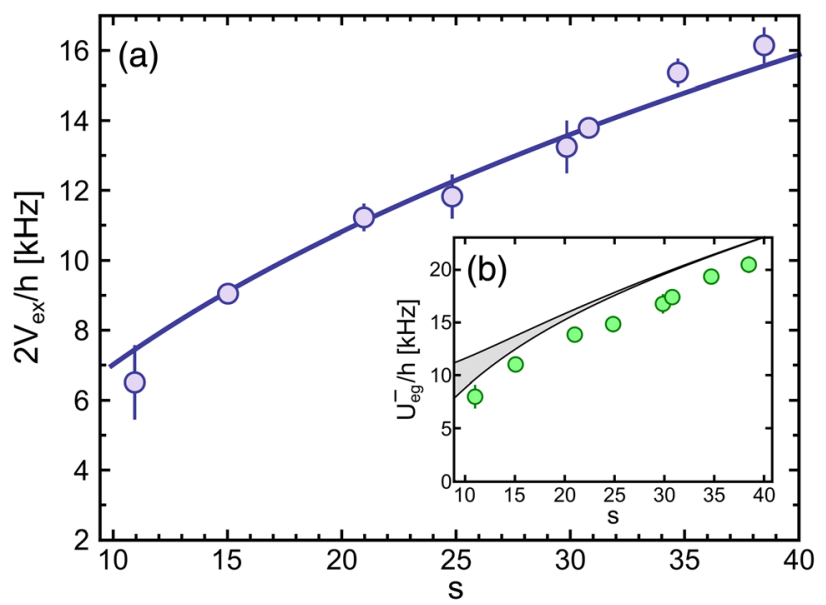

FIG. 3 (color online). (a) The points show the measured spinexchange frequency as a function of the lattice depth $s$. The data have been corrected for the small bias magnetic field $B=3.5 \mathrm{G}$ [15] in order to show the zero-field spin-exchange frequency. Each point is the average of at least three different measurements, and the error bar shows the statistical error. The line is a fit based on the model described in the main text. (b) The points show the interaction energy of the $\left|e g^{-}\right\rangle$state, calculated as the sum of the experimentally measured $2 V_{\text {ex }}$ and the $U_{e g}^{+}$calculated by using $a_{e g}^{+}=219.5 a_{0}$ [6]. The shaded area shows the energy difference between the ground and first excited lattice bands.

of two ground-state atoms trapped in the lattice sites, and approach from below the energy separation between the ground and first excited bands of the lattice. In this regime, the usual treatment of interactions, based on the evaluation of the Hubbard on-site interaction energy with the wellknown expression $U=\left(4 \pi \hbar^{2} a / m\right) \int|w(\mathbf{r})|^{4} d \mathbf{r}$ [where $w(\mathbf{r})$ are the single-particle Wannier functions], is expected to fail. At large interaction strength, the two-particle wave function cannot be expressed in terms of lowest-band Wannier functions since, in the limit of infinite repulsion, the two atoms tend to spatially separate in each lattice site [16] and the probability of finding them at the same position drops to 0 . For a system of two particles in a harmonic potential, it has been shown that, for a scattering length $a$ significantly larger than the harmonic oscillator length $a_{\mathrm{ho}}$, the interaction energy saturates at the energy of the first excited harmonic oscillator state $[17,18]$.

In order to relate our measurements to the values of the scattering lengths $a_{e g}^{ \pm}$, we follow a similar treatment to that adopted in Refs. [19,20], where the interaction energy for two particles in a true optical lattice potential was derived by evaluating the anharmonic corrections to the lowestorder parabolic approximation of the potential. In our analysis, we express the total Hamiltonian on a basis formed by wave functions for the relative motion and for the center-of-mass motion of the two particles. For the former, we use the wave functions for interacting particles in a harmonic trap analytically derived in Ref. [17]; for the latter, harmonic oscillator wave functions are considered (see the Supplemental Material [8] for more details). 
We then evaluate the anharmonic terms (up to tenth order) on this basis, and, by numerical diagonalization of the total Hamiltonian, we derive the dependence of the interaction energy in the motional ground state $U(a, s)$ as a function of the scattering length $a$ and of the lattice depth $s$. In Fig. 3(a), we fit the experimental data of the spin-oscillation frequency vs $s$ with the function $\left[U\left(a_{e g}^{-}, s\right)-U\left(a_{e g}^{+}, s\right)\right] / h$ (solid line), assuming the value $a_{e g}^{+}=219.5 a_{0}$ for the spinsinglet scattering length measured in Ref. [6] (where $a_{0}$ is the Bohr radius). The result of the fit is a spin-triplet scattering length $a_{e g}^{-}=(3300 \pm 300) a_{0}$. This scattering length is remarkably large and, as shown in Fig. 3(b), causes the energy of the $\left|e g^{-}\right\rangle$state to almost saturate to the energy gap between the first two lattice bands (grey curve).

At a finite $B$, the spin-exchange oscillation shows a faster frequency, as the Zeeman energy increasingly contributes to the energy difference between $\left|e g^{L}\right\rangle$ and $\left|e g^{H}\right\rangle$ (see Fig. 1). The circles in Fig. 4 show the measured spinoscillation frequency $\left(U_{e g}^{H}-U_{e g}^{L}\right) / h$ at $s=30$ as a function of $B$, while the squares indicate the energy of the $\left|e g^{L}\right\rangle$ state determined by fitting the position of the peaks in the spectroscopic measurements shown in Fig. 2(a). These data are compared to a simple single-band model in which the Hamiltonian of the two-atom system including interaction energy and Zeeman shift is written on the $\left\{\left|e g^{+}\right\rangle,\left|e g^{-}\right\rangle\right\}$ basis as

$$
H=\left(\begin{array}{cc}
U_{e g}^{+} & F \Delta_{B} \\
F \Delta_{B} & U_{e g}^{-}
\end{array}\right)
$$

where $\Delta_{B}=\Delta \mu B$ is the Zeeman splitting (arising from a difference $\Delta \mu$ in the magnetic moments of the $|g\rangle$ and $|e\rangle$

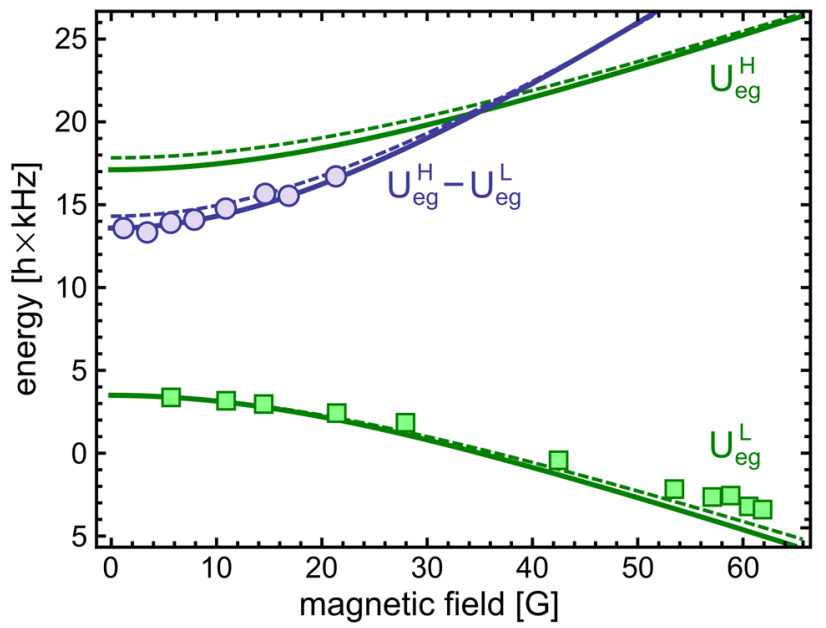

FIG. 4 (color online). The circles represent the measured spinexchange frequency $\left(U_{e g}^{H}-U_{e g}^{L}\right) / h$ at $s=30$ as a function of the magnetic field. The squares represent the measured energy of the $\left|e g^{L}\right\rangle$ state derived from the spectroscopic measurements exemplified in Fig. 2(a). The solid lines show the predictions of the model in Eq. (3) by using the $a_{e g}^{-}$value derived in Fig. 3. The dashed lines show a fit of the points to the same model, leaving $a_{e g}^{-}$as a free parameter (see the main text for more details). states [21]) coupling the zero-field eigenstates $\left|e g^{+}\right\rangle$and $\left|e g^{-}\right\rangle$. Differently from Ref. [6], we have included a Franck-Condon factor $F$, defined as the overlap integral

$$
F=\iint d \mathbf{r}_{1} d \mathbf{r}_{2} \psi_{e g}^{+*}\left(\mathbf{r}_{1}, \mathbf{r}_{2}\right) \psi_{e g}^{-}\left(\mathbf{r}_{1}, \mathbf{r}_{2}\right),
$$

between the wave functions $\psi_{e g}^{ \pm}$of the two atoms interacting in the two different channels. The strong repulsion in the spin-triplet channel causes indeed a strong modification of the wave function, resulting in an overlap integral that is significantly smaller than unity $(F \simeq 0.77$; see the Supplemental Material [8]). By diagonalizing Eq. (3), we find the eigenstates $\left\{\left|e g^{L}\right\rangle,\left|e g^{H}\right\rangle\right\}$ and the dependence of the energies $U_{e g}^{L}$ and $U_{e g}^{H}$ on the magnetic field $B$ (see also Fig. 1). The solid lines in Fig. 4 show the predictions of this model by using $a_{e g}^{+}=219.5 a_{0}$ and $a_{e g}^{-}=3300 a_{0}$ (from the fit in Fig. 3) and the $F$ factor calculated by using the interacting wave functions obtained previously. The agreement with the experimental data is quite good, showing the substantial validity of the model in Eq. (3) as long as the overlap factor $F$ between the interacting wave functions is considered. Alternatively, we have performed a simultaneous fit of the two data sets in Fig. 4 with the eigenenergies of Eq. (3) by expressing $U_{e g}^{-}$and $F$ as functions of the free parameter $a_{e g}^{-}$(obtained from the model discussed previously): the result (dashed lines) is $a_{e g}^{-}=(4700 \pm 700) a_{0}$, which is $\sim 2 \sigma$ away from the more precise determination coming from the fit of the data shown in Fig. 3. We note that a precise determination of $a_{e g}^{-}$is complicated by the fact that, in this regime of strong interactions, the dependence of $U_{e g}^{-}$on $a_{e g}^{-}$is extremely weak and small effects coming, e.g., from calibration uncertainties or from higher-order contributions in the theory could yield significant changes. We also note that in the presence of a tight trapping, the interpretation of the results in terms of an effective scattering length should be considered [22]. However, we stress that, differently from $a_{\text {eg }}^{-}$, our determination of $V_{\text {ex }}$ is free from any assumption or modeling and represents an accurate measurement of the spin-exchange coherent coupling in an actual experimental configuration.

The 3D lattice setting that we have used in our experiments has allowed us to study the dynamics of an isolated two-atom system in which only one atom is in the excited state, therefore significantly reducing the effects of inelastic $|e\rangle-|e\rangle$ collisions. Nevertheless, we measure a finite lifetime of the spin-exchange oscillations, on the order of $\sim 2 \mathrm{~ms}$, after which the oscillation amplitude becomes comparable with the scattering of the points [see the inset in Fig. 2(b)]. In order to investigate the origin of this damping, we have performed additional experiments in which we have introduced a variable waiting time $t_{\text {wait }}$ between the laser excitation to the $\left|e g^{L}\right\rangle$ state and the magnetic field quench. For $t_{\text {wait }}$ as large as $30 \mathrm{~ms}$ (more than 1 order of magnitude larger than the observed damping time), we still detect high-contrast spin-exchange oscillations. This rules out the 
explanation of the damping in the inset of Fig. 2(b) in terms of either a detrimental effect of inelastic $|g\rangle-|e\rangle$ collisions in doubly occupied sites or a possible collisional dephasing introduced by the tunneling of highly mobile atoms in excited lattice bands. After the exclusion of these fundamental mechanisms of decoherence, it seems highly plausible that the decay of the spin-exchange oscillations arises from technical imperfections (associated, e.g., with the fast switching of the magnetic field).

In conclusion, we have observed for the first time fast, long-lived interorbital spin-exchange oscillations by exploiting a system of ultracold AEL fermions trapped in a 3D optical lattice. The direct observation of several periods of these oscillations has allowed us to demonstrate the coherence of the process and to measure the exchange interaction strength in an accurate, model-independent way. We note that, if compared with the spin dynamics observed in other atomic systems, arising from either small differences in the scattering lengths [23-25] or from second-order tunneling between adjacent sites of an optical lattice [26], the oscillation that we have measured is significantly fast. In particular, the exchange energy $V_{\mathrm{ex}}$, on the order of $\sim h \times 10 \mathrm{kHz}$, is much larger than either the Fermi $\left(k_{B} T_{F}\right)$ or the thermal $\left(k_{B} T\right)$ energy, which makes ${ }^{173} \mathrm{Yb}$ remarkably interesting for the observation of quantum magnetism in a two-orbital system with $\mathrm{SU}(N)$ interaction symmetry [4]. The direct measurement of $V_{\mathrm{ex}}$ has also allowed us to provide a determination of the interorbital spin-triplet scattering length, which exceeds the spin-singlet one by $\sim 20$ times. Besides, from a wider point of view, this strong spin-exchange interaction entangles 2 stable internal degrees of freedom of the atom [27], which can be independently and coherently manipulated, opening new realistic possibilities for both quantum information processing and quantum simulation.

We would like to acknowledge N. Fabbri, M. Fattori, C. Fort, and A. Simoni for useful discussions, F. Schäfer for early experimental contributions, and Qubig $\mathrm{GmbH}$ for valuable support in the initial testing phase of the narrow laser setup. This work has been financially supported by EU FP7 Projects SIQS (Grant No. 600645) and SOC-2 (Grant No. 263500), MIUR Project PRIN2012 AQUASIM, and ERC Advanced Grant DISQUA (Grant No. 247371).

*Corresponding author.

fallani@lens.unifi.it

[1] M. Inguscio and L. Fallani, Atomic Physics: Precise Measurements and Ultracold Matter (Oxford University Press, New York, 2013).

[2] S. Taie, R. Yamazaki, S. Sugawa, and Y. Takahashi, Nature Phys. 8, 825 (2012).

[3] G. Pagano et al., Nature Phys. 10, 198 (2014).

[4] A. V. Gorshkov, M. Hermele, V. Gurarie, C. Xu, P. S. Julienne, J. Ye, P. Zoller, E. Demler, M. D. Lukin, and A. M. Rey, Nature Phys. 6, 289 (2010).
[5] X. Zhang et al., arXiv:1403.2964.

[6] F. Scazza et al., arXiv:1403.4761.

[7] In the notation $|g \uparrow, e \downarrow\rangle$, the two-particle exchange symmetry is implicit. The full state has to be intended as $|g \uparrow, e \downarrow\rangle=\left(|g \uparrow\rangle_{1}|e \downarrow\rangle_{2}-|e \downarrow\rangle_{1}|g \uparrow\rangle_{2}\right) / \sqrt{2}$, which is antisymmetric for the exchange of fermion 1 with fermion 2.

[8] See Supplemental Material at http://link.aps.org/ supplemental/10.1103/PhysRevLett.113.120402, which includes Refs. $[9,10]$, for additional technical details on the experimental procedure and on the theoretical model.

[9] D. Jaksch, C. Bruder, J. I. Cirac, C. W. Gardiner, and P. Zoller, Phys. Rev. Lett. 81, 3108 (1998).

[10] J. L. Hall and M. Zhu, in Proceedings of the International School of Physics "Enrico Fermi," Course CXVIII, edited by E. Arimondo, W. D. Phillips, and F. Strumia (NorthHolland, Amsterdam, 1992), p. 686.

[11] M. Pizzocaro, G. A. Costanzo, A. Godone, F. Levi, A. Mura, M. Zoppi, and D. Calonico, IEEE Trans. Ultrason. Ferroelectr. Freq. Control 59, 426 (2012).

[12] Z. Barber et al., Phys. Rev. Lett. 100, 103002 (2008).

[13] In the limit $t_{\text {ramp }} \rightarrow 0$, the relative population of the $\left|e g^{-}\right\rangle$ and $\left|e g^{+}\right\rangle$states would be $\beta^{2} / \alpha^{2}$.

[14] T. Sleator, T. Pfau, V. Balykin, O. Carnal, and J. Mlynek, Phys. Rev. Lett. 68, 1996 (1992).

[15] We note that the finite bias magnetic field $B \simeq 3.5 \mathrm{G}$ used for the measurement yields a slightly faster oscillation frequency than $2 V_{\mathrm{ex}} / h$ (by $\sim 100 \mathrm{~Hz}$ ). In order to show the zero-field oscillation frequency, the data points in Fig. 3 have been corrected by using the finite- $B$ model described later in the text.

[16] G. Zürn, F. Serwane, T. Lompe, A. N. Wenz, M. G. Ries, J. E. Bohn, and S. Jochim, Phys. Rev. Lett. 108, 075303 (2012).

[17] T. Busch, B.-G. Englert, K. Rzażewski, and M. Wilkens, Found. Phys. 28, 549 (1998).

[18] M. Köhl, K. Günter, T. Stöferle, H. Moritz, and T. Esslinger, J. Phys. B 39, S47 (2006).

[19] F. Deuretzbacher, K. Plassmeier, D. Pfannkuche, F. Werner, C. Ospelkaus, S. Ospelkaus, K. Sengstock, and K. Bongs, Phys. Rev. A 77, 032726 (2008).

[20] J. Mentink and S. Kokkelmans, Phys. Rev. A 79, 032709 (2009).

[21] S. G. Porsev, A. Derevianko, and E. N. Fortson, Phys. Rev. A 69, 021403(R) (2004).

[22] E. L. Bolda, E. Tiesinga, and P. S. Julienne, Phys. Rev. A 66, 013403 (2002).

[23] A. Widera, F. Gerbier, S. Fölling, T. Gericke, O. Mandel, and I. Bloch, Phys. Rev. Lett. 95, 190405 (2005).

[24] J. S. Krauser, J. Heinze, N. Fläschner, S. Götze, O. Jürgensen, D.-S. Lühmann, C. Becker, and K. Sengstock, Nature Phys. 8, 813 (2012).

[25] J. S. Krauser, U. Ebling, N. Fläschner, J. Heinze, K. Sengstock, M. Lewenstein, A. Eckardt, and C. Becker, Science 343, 157 (2014).

[26] S. Trotzky, P. Cheinet, S. Fölling, M. Feld, U. Schnorrberger, A. M. Rey, A. Polkovnikov, E. A. Demler, M. D. Lukin, and I. Bloch, Science 319, 295 (2008).

[27] M. Anderlini, P. J. Lee, B. L. Brown, J. Sebby-Strabley, W. D. Phillips, and J. V. Porto, Nature (London) 448, 452 (2007). 